\title{
Virtual Reality Art Visualised Through Surface Relief Digital Holography
}

\author{
Ioana Pioaru \\ Wrexham Glyndŵr University \\ Mold Rd, Wrexham LL11 2AW, UK \\ ioa photodendron@yahoo.com
}

\begin{abstract}
This paper proposes surface relief digital (SRD) holography as a method for visualising VR art for the purpose of presentation in a fine art context. VR holography is a new art form, which synthesizes the qualities of traditional hand-drawing with the unique features of holography and virtual reality art. Previous projects addressing the topic of VR holography have demonstrated how direct-write (reflection) digital holography can be used successfully to display artworks generated through VR art-making tools such as Tilt Brush, Gravity Sketch etc., but have also emphasised the limitations of this medium. SRD holography offers a solution to some of these limitations, namely the relatively low brightness characteristic of reflection holography and difficulties related to the preservation of artworks recorded on photographic materials suitable for reflection holography. SRD transmission holograms are significantly brighter than reflection holograms due to their intrinsically more efficient use of the illumination light. Also, SRD holography is an outstanding medium from the point of view of archivability - the fact that SRD holograms are printed on glass and the nature of the photoresist coating itself makes these holograms fully archival. They are therefore much easier to preserve than silver halide film holograms or even dichromate gelatine glass holograms, which are prone to humidity-related damage even when great care is taken in sealing them. The SRD transmission holograms presented in this paper have been printed with a high-resolution digital printer and have a hogel size of $\mathbf{1 0 0}$ microns - practically imperceptible to the naked eye. By contrast, VR holograms presented in previous studies were printed with a hogel size of $0.8 \mathrm{~mm}$. This leads to a visible pixelation, which constitutes a distracting feature when this technique is used in a fine art context. Finally, SRD holograms exhibit the unique property that they can be seen from both sides without any loss in quality (when displayed on plinths for example), something that substantially increases the number of people who can view the artworks simultaneously.
\end{abstract}

Virtual reality art. Holography. Surface relief digital holography. Virtual reality holography.

\section{INTRODUCTION}

In recent times, VR artmaking has become increasingly popular among artists. Applications such as Tilt Brush or Gravity Sketch are impressive tools for creating digital 3D imagery, offering great new possibilities for art and the creative industries. However, the high cost of purchasing a VR system and also the space required to run it are prohibitive factors, which still hinder the spread of this medium. In addition, artworks created in VR are fundamentally difficult to view or exhibit outside of virtual space without losing some of their essential features. Online galleries, which allow the viewer to explore VR projects using a standard monitor, flatten the three-dimensionality of VR artworks, as is the case with any $2 \mathrm{D}$ representation of a volume (e.g. The Google Tilt Brush AIR gallery https://www.tiltbrush.com/air/artists/). Attempts have been made to use $3 \mathrm{D}$ printing as a means to visualise VR art, but due to its own constraints, 3D printing only allows for a small range of VR models to be transformed into real objects (Fantasygraph 2016).

The advantage of bringing together VR and holography, which I started to do in 2017, is that it allows for an artwork produced in VR to be displayed and viewed easily in either a public gallery or a private space. A larger number of people can experience the work simultaneously, without having to wear a cumbersome headset in order to perceive the three-dimensional appearance. My early projects with this hybrid medium have shown how digital holography can offer a high fidelity of representation, and is able to preserve the properties of VR imagery (Pioaru 2017, 2018). 
The principle reason for starting to work with VR and holography was my interest in expanding the possibilities of artistic expression intrinsic to drawing by exploring drawing through different media. Being very new to holography, it was never my intention to master the technical aspects of the medium, since this requires a great amount of time and almost exclusive dedication to this endeavour. This is demonstrated by practitioners who devoted their careers to working with holography, undertaking the difficult task of making their own holograms (e.g. Benyon). Instead, I chose to leave the technicalities to the holography experts working at Geola while I focused on the content of the artworks and on issues related to their presentation in an art gallery context. At the beginning, I found the limitations inherent to holography rather daunting. The lack of brightness and the unstable, perishable support material were particularly problematic.

Trying to find a solution to these issues led my investigations towards the use of SRDH. Firstly, I wanted to test out the idea of drawing with white lines on a black background, as a means to tackle the brightness issue. Secondly, as I discovered that the SRD hologram service offered by Geola used glass plates coated with photoresist (rather than the silver halide film used for refection holograms), I was optimistic that this might be a solution to the problem of archivability.

\subsection{VR holography and maximalism}

My explorations of VRH are a part of my ongoing doctoral research around the concept of maximalism. Not strictly the opposite of minimalism but more easily understood as such, maximalism is defined as a type of practice that engages with the 'upper limits' of artistic expression; maximalist tendencies can be manifested in a variety of ways, for example spatial maximalism designates a type of art whose dominant feature is quantitative expansion: it makes a point of either taking up as much space as possible, taking up more space than is usually understood as 'the space of art', infiltrating into the liminal territories of the non- or para-artistic, or expanding the physical space of art by inhabiting the world of the virtual, as in the case of digital art and virtual and augmented reality art. Holography constitutes a unique instance of spatial maximalism in that it expands space in an illusory fashion - holographic objects do not actually take up the volume of space, which they appear to occupy. Holographic art is thus inherently maximalist as it contains (or simulates) an extra dimension - that of volume - which is not physically present in the artwork but is instantiated in the meeting of the artwork, the light, and the viewer.

\section{EARLY ATTEMPTS}

This section briefly presents the VRH projects I have developed between 2017 and 2019 before starting to use the SRDH technique.

\subsection{Van Gogh by Pioaru, 2017}

In 2017 I tried for the first time to use holography in conjunction with VR art (Pioaru 2017). The VR project was created using Google Tilt Brush and an HTC Vive headset and, since it was a reinterpretation of a Van Gogh self-portrait' I chose the title Van Gogh by Pioaru.

Part of the process of transferring the VR project to holography entails exporting the VR scene as an fbx file, which is then used in a 3D software (in this case 3DS Max) to generate the image data required by the digital holographic printer; however, due to various software incompatibilities, only the volumes were imported from TB into 3DS Max, while the colour and texture information was lost. This meant that the scene had to be recoloured in 3DS Max. In addition, when imported, the brush strokes were reduced to plain, ribbon-like meshes. All this resulted in significant differences between the original VR scene and the final reflection hologram, which was printed from my data set using a commercial digital holographic printer (Figure 1).

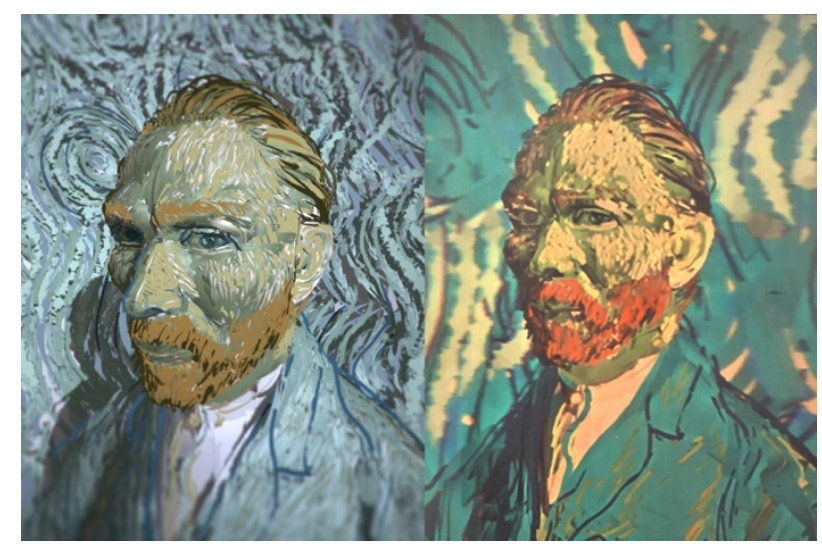

Figure 1: A snapshot taken in Tilt Brush (left) and a photograph of the final hologram (right)

\subsection{Spectral Figures, 2018 (ongoing)}

Notwithstanding the technical difficulties encountered, Van Gogh by Pioaru was an encouraging first step. It had been well received in the context of an international conference (Cyberworlds 2017) and it confirmed the possibility to display VR images through digital holography; but the obstacles prompted by this first experiment determined me to adapt my next project, Spectral Figures (Pioaru 2018), to the limitations of the transfer process. The next project consisted of a series of black and white portraits of artists and 


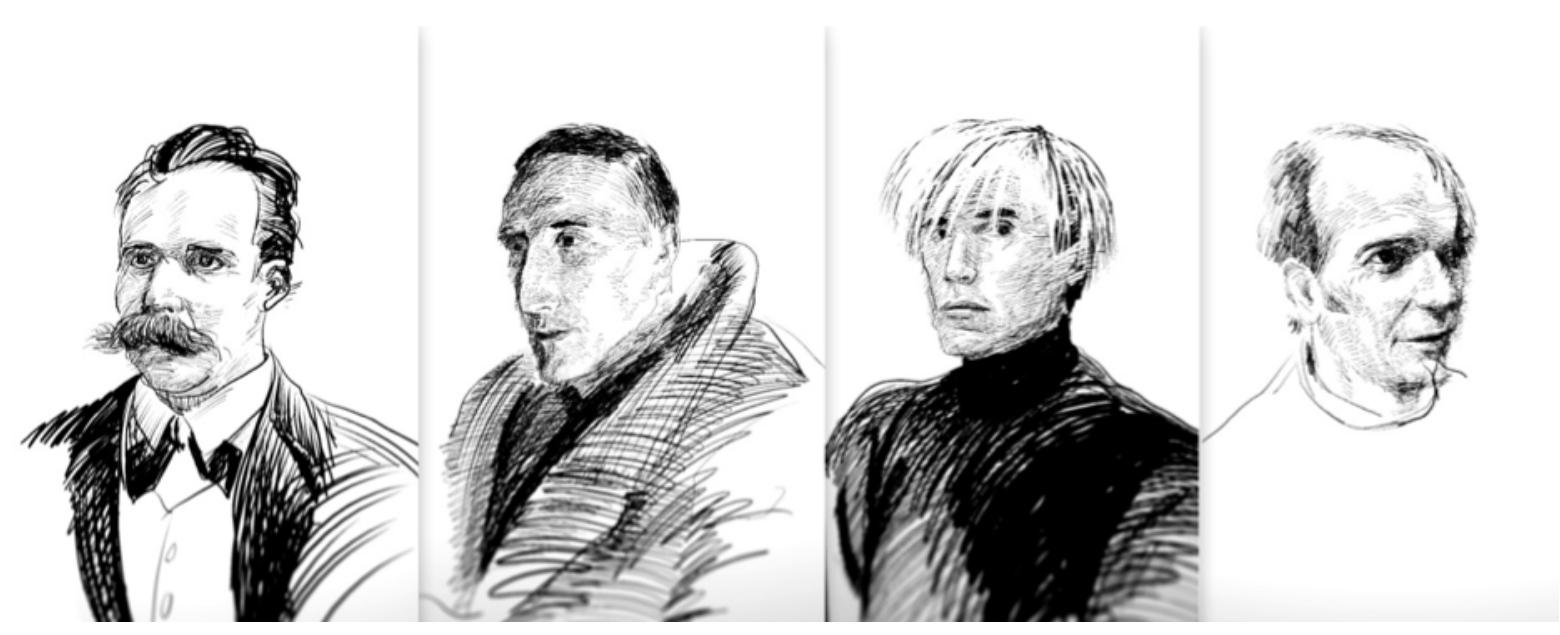

Figure 2: 'Spectral Figures' series - a collage of snapshots taken in Tilt Brush.

philosophers whom I consider to be influential to my practice. I chose to draw them in black and white in order to reduce the risks associated with recolouring the scene. I used thin black strokes set against a white background to emulate the characteristics of drawing on paper. As a consequence, possibly the most striking aspect of the resulting images is that, although effectively they are sculptures, every viewing angle gives the illusion of a $2 \mathrm{D}$ line drawing.

The decision to restrain the use of the available VR tools, albeit a compromise, brought me a step closer to one of my preferred artistic media, i.e. drawing, and particularly to 'sculptural drawing'; this is a concept which I have addressed in some of my recent projects and it refers to expanding the characteristics of drawing to create sculptural objects, as part of my interest in exploring the boundaries between $2 d$ and $3 d$ imagery.

The image data was sent to Geola for printing on their digital holographic printer and a series of $30 \mathrm{~cm} \times 40 \mathrm{~cm}$ full-parallax, full-colour reflection holograms was produced using a silver halide emulsion. The holograms, when properly illuminated, produced a good impression of the original VR subject.
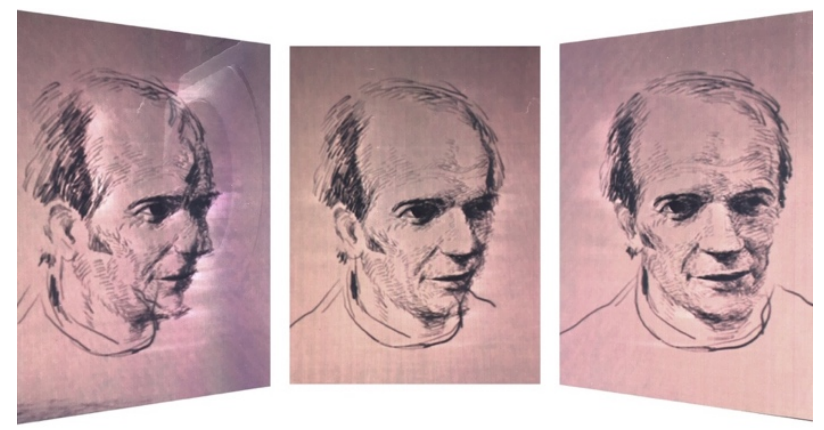

Figure 3: Three views of 'Spectral Figures \#4', fullparallax reflection hologram.

\subsection{Tilgate Forest, 2019}

For this new project inspired by one of my recent ink drawings, I used the same tools as I did in Spectral Figures, i.e. black strokes on a white background, but I wanted to explore a theme that was different from my previous VRH art works, which had been exclusively portraits. Since the landscape is a rather underrepresented genre in holography, it promised to produce an interesting result.

While drawing the scene in VR, I distorted the composition by making objects in the distance much smaller and closer to the main viewpoint than they would be in an accurate scale model of the real landscape, as in a diorama, in order to limit the angular resolution required to resolve the rearmost parts of the scene. The resulting hologram was close enough to the original ink drawing and quite convincing as a 3D depiction of a landscape, in terms of parallax effect. However, pure black and white images such as Spectral Figures and Tilgate Forest represent particularly challenging subjects for holography, straining its capabilities with respect to brightness and colour balance. Additionally, while the maximum angular resolution of digital holography itself is state of the art (thousands of views), current replay illumination techniques reduce this by an order of magnitude. As a result, although the diorama technique used in Tilgate Forest was an effective illusion in its own right, it was insufficient to fully mitigate the blurring imposed on the background of the scene. This somewhat compromised the pen-drawing appearance, the very fine brush strokes seemingly blending together to create a charcoal-like effect (Pioaru \& Stokes 2019). 

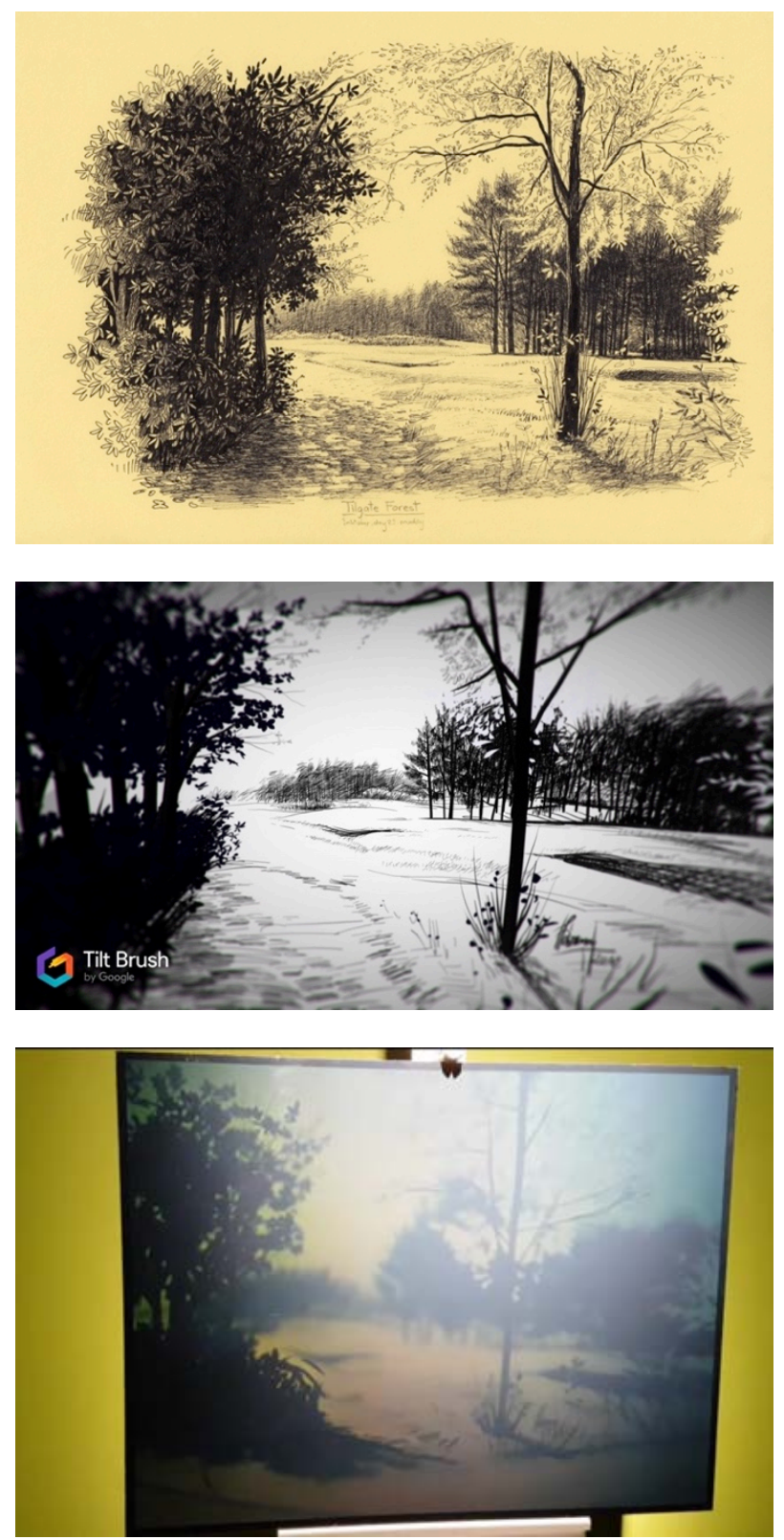

Figure 4: Three Tilgate Forest images: ink drawing (top), VR drawing (centre), full-parallax reflection hologram (bottom).

\section{THE PROCESS OF MAKING AN SRD HOLOGRAM: SPECTRAL FIGURES - SEA TURTLE}

SRDH offers a solution to some of the limitations mentioned above. SRD transmission holograms are in general significantly brighter than the reflection holograms presented above, due to their intrinsically more efficient use of the illumination light. SRDH is an outstanding medium from the point of view of archivability as it is based on a glass rather than film substrate. The SRD holograms presented here were printed on a digital holographic printer at Geola using a hogel size of 100 microns - practically imperceptible to the naked eye, which solves the hogelisation (visual pixelation caused by larger hogel sizes) issue and using a relatively limited horizontal field of view of $+/-30$ degrees, which again increases final image brightness.

To illustrate the process of making a VR hologram using the SRDH technique, I present one of my recent projects titled Spectral Figures: Sea Turtle, part of a series of artworks depicting critically endangered species of animals.

When making a VR project intended for transfer onto holographic format, one needs to bear in mind not just the limitations of holography but also the problems incurred by the transfer process itself, which principally come down to the incompatibility between VR art-making software (such as Tilt Brush) and other 3D modelling software (e.g. 3DS Max or Blender). The fact that, at the moment, critical data is lost in the transfer process when using the fbx export file limits the range of tools which an artist can use in Tilt Brush. It is likely that these shortcomings will soon be resolved. One step in this direction has already been taken - the development of a Tilt Brush toolkit destined for Unity which allows for all content, including special effects, to be successfully imported. Similar toolkits for a variety of digital creation software would significantly ease the transfer process. Meanwhile, in the absence of these tools, I was determined to develop a pipeline to work around these issues. The process presented in this section was similar for all of my VRH projects from the beginning, but it was refined after each project, culminating with my most recent SRD holograms.

The first step after choosing a subject is 'understanding the volume', i.e. familiarising oneself with the appearance and proportions of the subject through a series of preliminary 2D sketches. I personally consider this stage to be very important, as I am interested in the likeness between the subject and the VR sculptural drawing rendition, but it is by no means essential to the process in general - it is a matter of personal preference and approach to art-making.

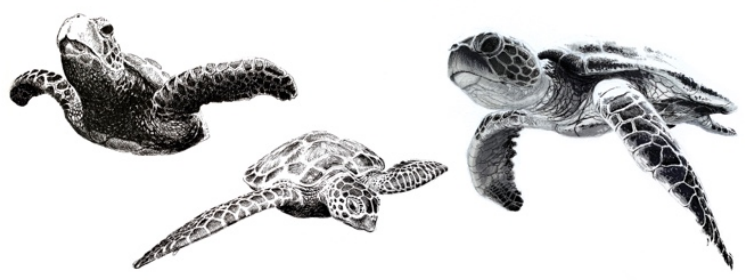

Figure 5: Preparatory ink sketches for 'Spectral Figures: Sea Turtle'.

Some of these sketches are then imported into the VR environment and are used as a reference, in a similar way as one would draw after a model or 
'from life', in real space. The difference is that, while in real-space drawing the $3 d$ information from the model is compressed into a flat $2 d$ rendition of it, in virtual space the visual information from the various $2 d$ sketches is corroborated to create a $3 d$ structure or, as I call it, a sculptural drawing.

When constructing my VR sculptural drawing, bearing in mind that that the scene will need to be recoloured, I am careful to pair colours with brushes, meaning that I always use the same brush for a certain colour. There are some Tilt Brush drawing tools which are exported as volumes, and other that are exported as meshes. The latter usually look very different when exported - for example a brush which produces stylised, tapered, textured strokes in TB, will appear as a flat rectangular mesh in the export. Therefore, I have a preference for the former, which are more predictable as they tend to maintain the same volumetric appearance.

When the VR project is finished, I take a snapshot of it with the inbuilt camera from roughly the same perspective as the hologram main viewpoint - this will later help to set up the camera which will generate the image data for the holographic printer.

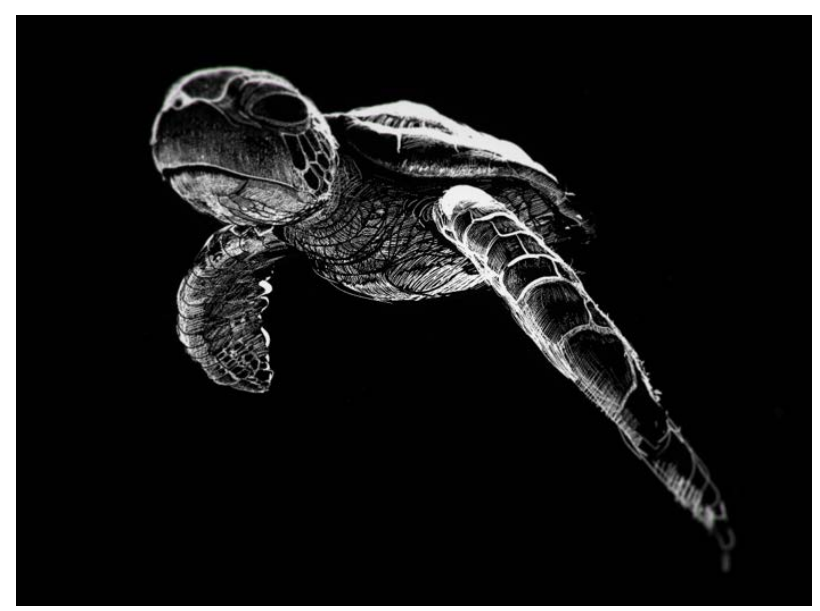

Figure 6: Snapshot of the VR version of 'Spectral Figures: Sea Turtle', taken in Tilt Brush.

After deleting the reference images, I export the project and then open the resulting fbx file with Blender. The next stages of the process are done in collaboration with Geola who are currently developing a tool for automating this postprocessing phase so as to enable artists to easily prepare the file for being printed. Briefly, this consists of reapplying the correct colours to the volumes, setting up the 'holoplane' (a virtual plane that simulates the holographic plate) and positioning an animated camera at the correct distance from the holoplane using a method described by Brotherton-Ratcliffe and Bjelkhagen (2012).
The data produced by the Blender camera is for a single parallax, $150 \mathrm{~mm} \times 150 \mathrm{~mm}$, SRD hologram with a hogel size of 100 microns, printed on glass sheets coated with photoresist, using a DWDH transmission printer with a blue pulsed laser. The hologram can be replayed with an array of either halogen or diode lights. The advantage of the array is that they increase the angle from which the drawing appears to be black \& white at the same time as increasing brightness. Outside this angle, the appearance is that of a rainbow hologram.
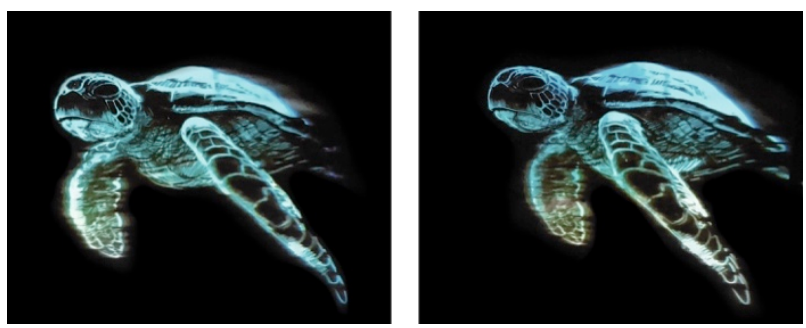

Figure 7: 'Spectral Figures: Sea Turtle': two views of the final SRD hologram.

\section{RESULTS: SRD HOLOGRAMS VS. REFLECTION HOLOGRAMS}

The resulting SRD holograms are superior to the previous reflection holograms in terms of visual quality.

The technique of drawing using a white pen with a black background effectively routes the light energy available from the hologram into the drawing lines; and since the drawing lines usually occupy a much smaller solid angle in total than the background, the relative brightness of the lines can be much higher. This is a technique which, in principle, could also be used with reflection holography, except that the usually rather larger hogel sizes characteristic of reflection hologram printers do not permit the use of such fine lines as current SRD printers allow.

Another effect of this technique of "white on black" is that when one switches on the light illuminating the hologram, the only thing which changes is that the drawing appears in front of a black (invisible) background. In contrast, when drawing with a black pen on a white background, the whole background changes from black to white as the light is turned on.

A further advantage of this technique is that hogelisation is far less perceptible through the bright pen lines as compared to their perceptibility through a white background. White is also the hardest colour to achieve in holography and large areas of white tend to show up discolouration all too often. 
Due to the small hogel size of the SRD holograms produced, the images appear substantially crisper than the reflection VR holograms reviewed above. Hogelisation is a distracting feature in the context of VR holographic art. It is a particular problem with full-parallax holograms, which, through their nature, invite the viewer to look at the artwork from all distances including from very close up. Currently Geola offers a $0.8 \mathrm{~mm}$ hogel with its DWDH reflection film printers. Although in principle it is possible to make smaller hogels with reflection holograms, it is much easier and practical with SRD holography. This is due to a variety of reasons, the most important of which are the ability of SRD holography to withstand brightness erosion due to the smaller hogel and the compatibility of photoresist with pulsed lasers. Pulsed lasers allow high-speed printing of DWDH holograms, including SRD holograms, and one must bear in mind that a 100-micron hogel hologram takes 64 times longer to print than a $0.8 \mathrm{~mm}$ hogel hologram with the same laser repetition rate. When a photographic material is not compatible with pulsed laser radiation, write time increases by up to 30 to even 100 times, meaning that practically ultra-small hogel non-SRD holograms are not currently possible.

A further advantage of SRD single-parallax holograms is that the digital image data is usually some hundreds of times smaller than the data required for full-parallax holograms. This means that whilst single parallax holograms can be calculated on a normal laptop or PC, full-parallax holograms often need either a reasonably large network of PCs or a small supercomputer.

Perhaps most important of all is that SRD holography offers an excellent solution to the problem of archivability - a critical aspect in the context of fine art presentations, as the durability and the quality of materials is often one of the rigors imposed on the artwork. The fact that SRD holograms are printed on glass and the nature of the photoresist coating itself makes these holograms fully archival. As such, they are much easier to preserve than silver halide film holograms or even dichromate gelatine glass holograms, which are prone to humidity-related damage even when great care is taken in sealing them.

A peculiarity of this type of hologram is that it is visible both in reflection and in transmission equally well, which doubles the number of people who can view the artworks simultaneously.

The principle disadvantage of SRD holography is that it can only produce single-parallax images. From an absolute point of view the full-parallax hologram is, of course, to be preferred as it presents the most general and most faithful $3 d$ experience of the original light-field to the viewer. Single-parallax holograms remove the vertical parallax from the light-field. As the viewer moves his head up and down, the 3d image will appear to simply tilt up and down. Since our eyes are horizontally separated, this type of hologram still looks very realistic to most of us. The tilting of the image is fairly well accepted by the viewer and interpreted as a natural movement of the image itself. The largest drawback with single-parallax holograms is that the viewer cannot approach too closely the hologram without image distortion becoming excessive.

A disadvantage that should also be mentioned is related to my choice of using a 100-micron hogel. As explained, this is well tolerated by SRDH and has many advantages; one disadvantage however occurs due to a phenomenon called digital diffractive blurring (Brotherton-Ratcliffe \& Bjelkhagen 2012). The slight blurring that can be seen in the turtle's front limbs (particularly the one in the distance) is a drawback of the small hogel size caused by diffractive blurring. The clarity of an SRD hologram near the hologram plane is inversely proportional to the hogel size. However, the depth of field (which measures that distance around the image plane which remains in focus) increases with the hogel size. As such, an increase in the hogel size from 100 microns to 200 microns for instance would resolve the blurring issue by increasing depth of field, but the image would appear slightly more pixelated around the image plane. To have 'the best of both worlds', an artist using this technique therefore has to concentrate most of the detailed imagery quite close to the holoplane.

The reduced angle of view present in Spectral Figures: Sea Turtle is a peculiarity of the Geola SRD printer. It is worth mentioning that this was the first time it was used for printing an artwork - the primary use of this printer is the production of embossed holograms on metal shims for various commercial and security applications.

\section{CONCLUSIONS}

As mentioned before, the focus of this study is to assess the qualities of SRD holography from a fine art perspective and not strictly to judge its capacity to perfectly reproduce a VR project.

The primary conclusion of this paper is that, despite a few constraints such as the reduced angle of view (a peculiarity of the Geola printer I used, not of the SRDH method in general) and the loss of vertical parallax, the advantages enumerated above make SRDH very suitable for use as an art medium in conjunction with VR imagery. 
Developments in illumination techniques, for example the possibility of using linear light sources for replaying SRD holograms, promise to further enhance the quality of these artworks.

VR holography is currently the only medium that preserves the three-dimensional appearance of virtual works and affords simultaneous group viewing without equipment such as glasses or head-mounted devices - an important consideration in the context of an art gallery and $\mathrm{SRDH}$ is an important step forward in terms of visual quality.

Artists willing to explore VR SRDH should therefore be encouraged to consider its flaws as simply an inherent limit of the medium. Understanding how to adapt to the specificity of a certain technique and how to use it to its best effect constitutes a challenge, which is common to all artistic media. Perhaps the most significant difference is the highly technical nature of holography and the burden that this places on the artist, given the scarcity of holographic facilities available around the world.

In September 2019, a selection of my VR SRD holograms was included in a group exhibition, 'Holographic Embodiment', organised at the Centre for the Holographic Arts in New York. On this occasion as well as previous public presentations of $\mathrm{VRH}$, the work was well received both by the general public and by artists and scientists attending the event and was deemed highly innovative, particularly the idea and method of combining traditional drawing with the medium of digital holography.

The next envisaged step is to present VR SRDH artworks in art galleries and venues other than those interested primarily in holographic art. Here, they could be brought to the attention of not only a wider audience but also art experts who could assess their relevance within the landscape of contemporary art. Hopefully, the dissemination of $\mathrm{VRH}$ artworks would then result in more art practitioners deciding to work with it and finding it stimulating, useful, and inspiring.

\section{REFERENCES}

Benyon, M. (1994) How is holography Art? PhD Thesis

https://ethos.bl.uk/OrderDetails.do?uin=uk.bl.ethos. 359209 (retrieved 16 March 2020)

Bjelkhagen, $H$. and Brotherton-Ratcliffe, D. (2013) Ultra-realistic imaging: Advanced Techniques in Analogue and Digital Colour Holography, Boca Raton, London, New York: CRC Press, Taylor \& Francis Group, 357-368; 420-421.

Fantasygraph (2016) Three rules to avoid support structures when 3D printing. https://pinshape.com/blog/dotm-fantasy-graph-3dprinter-support-structures/ (retrieved 16 March 2020).

Geola (2020) Geola Digital UAB - optics and lasers manufacturing and research company based in Vilnius, Lithuania, established in 1995. http://geola.com (retrieved 16 March 2020).

Pioaru, I. (2017) Visualizing virtual reality imagery through digital holography. International Conference on Cyberworlds (CW 2017), Chester, United Kingdom, 20-22 September 2017, 241-244. doi:10.1109/CW.2017.50

Pioaru, I. (2018) From virtual reality to digital holography, International Symposium on Display Holography (ISDH 2018), Aveiro, Portugal, 25-29 June 2018, 49-52.

Pioaru, I. and Stokes, D. (2019) 'A comparative analysis of virtual-reality art-making software for the production of VR digital holograms. SPIE OPTO: Practical Holography XXXIII: Displays, Materials, and Applications (SPIE 2019), San Francisco, California USA. doi: 10.1117/12.2512233.

Tiltbrush Artist in Residence Gallery (2020). https://www.tiltbrush.com/air/artists (retrieved 12 March 2020) 